\title{
A New Adaptive Probabilistic Model of Blood Vessels for Segmenting MRA Images
}

\author{
Ayman El-Baz ${ }^{1}$, Aly A. Farag ${ }^{1}$, Georgy Gimel'farb ${ }^{2}$, Mohamed A. El-Ghar ${ }^{3}$, \\ and Tarek Eldiasty ${ }^{3}$ \\ ${ }^{1}$ Computer Vision and Image Processing Laboratory, \\ University of Louisville, Louisville, KY 40292 \\ \{elbaz, farag\}@cvip.Louisville.edu \\ http://www.cvip.louisville.edu. \\ ${ }^{2}$ Department of Computer Science, Tamaki Campus, \\ University of Auckland, Auckland, New Zealand \\ ${ }^{3}$ Urology and Nephrology Department, \\ University of Mansoura, Mansoura, Egypt
}

\begin{abstract}
A new physically justified adaptive probabilistic model of blood vessels on magnetic resonance angiography (MRA) images is proposed. The model accounts for both laminar (for normal subjects) and turbulent blood flow (in abnormal cases like anemia or stenosis) and results in a fast algorithm for extracting a 3D cerebrovascular system from the MRA data. Experiments with synthetic and 50 real data sets confirm the high accuracy of the proposed approach.
\end{abstract}

\section{Introduction}

Accurate cerebrovascular segmentation using non-invasive MRA is a valuable tool for early diagnostics and timely treatment of intracranial vascular diseases. Among three common MRA techniques, such as time-of-flight MRA (TOFMRA), phase contrast angiography (PCA), and contrast enhanced MRA (CEMRA). Today's most popular techniques for segmenting blood vessels from MRA data can be roughly classified in two categories: deformable models and statistical methods. The former iteratively adjust an initial boundary surface to blood vessels by optimizing an energy function that depends on image gradient and surface smoothness. Topologically adaptable surfaces make classical deformable models more efficient in segmenting intracranial vasculature [1]. Geodesic active contours implemented with level set techniques offer flexible topological adaptability to segment MRA images [2] including more efficient adaptation to local geometric structures represented e.g. by tensor eigenvalues [3]. Fast segmentation of blood vessel surfaces is obtained by inflating a 3D balloon with fast marching methods [4]. In 5], the capillary action of the blood inside the arteries and veins was modelled and used as an external force for the deformable model to segment the blood vessels. Several methods have been developed to segment the blood vessels based on extraction of skeleton of blood vessels and using multiscale schemes to allow for the diversity of vessel sizes 677. In these approaches, the 
centerline models can be generated explicitly, implicitly or via postprocessing by vessel modelling methods.

The MRA images are multi-modal in the sense that particular modes of the marginal probability distribution of signals are associated with regions-ofinterest. To the best of our knowledge, the only adaptive statistical approaches for extracting blood vessels from the MRA data were proposed by Noble and her group [89]. The marginal distribution is modeled with a mixture of two Gaussian and one uniform or Rician components for the stationary CSF (cerebrospinal fluid) and bones, brain tissues (white and gray matter), and arteries, respectively. The uniform component presumes the blood flow is strictly laminar. The mixture is identified (estimated) with a conventional EM algorithm.

Our paper derives a more general probability model of blood vessels on MRA images to account for normal and abnormal states of the vascular system, i.e. for both laminar and turbulent blood flow without and with stenosis. To accurately separate blood vessels from other regions-of-interest, the marginal distribution is precisely approximated with an adaptive linear combination of the derived model and a number of dominant and subordinate discrete Gaussians rather than with a mixture of only three pre-selected Gaussian and uniform or Rician components. Experiments show that our adaptive model results in significantly better segmentation of MRA images.

\section{Probability Model of Vascular Signals}

Let $q ; q \in \mathbf{Q}=\{0,1, \ldots, Q-1\}$, be the $Q$-ary signals (image intensity, or gray level). Conventional models of intensities for vessel voxels in [8]9] assume laminar blood flow with parabolic velocity flow through a circular cross-section of the vessel [10]. Then the intensity profile for a vessel is $q_{r}=q_{\max }\left(1-\frac{r^{2}}{R^{2}}\right)$ where $q_{r}$ is the intensity at the distance $r$ from the center of a vessel of radius $R$ and the constant $q_{\max } \leq Q-1$ depends on scanner. In this case the intensities over the circular cross-section are distributed uniformly with the probability density: $\varphi_{\text {lam }}(q)=\frac{1}{q_{\max }}$ in the range $\left[0, q_{\max }\right]$. Nonetheless, the laminar flow holds only for subjects with normal vascular systems [11.

Various diseases change either blood velocity or viscosity or both and cause the turbulent flow. Turbulence depends on the diameter of vessel and blood velocity and viscosity. Due to lower blood viscosity, anemia leads frequently to turbulence. Artery constrictions increasing blood velocity (see Fig. 1) and vascular diseases such as thrombosis, embolism, thyrotoxicosis, atherosclerosis, and valvular heart diseases also result in turbulence [11.

Typically, the turbulence adds a uniform random factor $\xi$ in the range $[-1,1]$ to the parabolic intensity profile [11:

$$
q_{r}=q_{\max }\left(1-\xi^{2} \frac{r^{2}}{R^{2}}\right)
$$

To derive the probability density of the intensities in Eq. (11) over the vessel, let $a_{r}=\pi r^{2}$ and $a_{\max }=\pi R^{2}$ be a circular area of radius $r$ and the maximum 

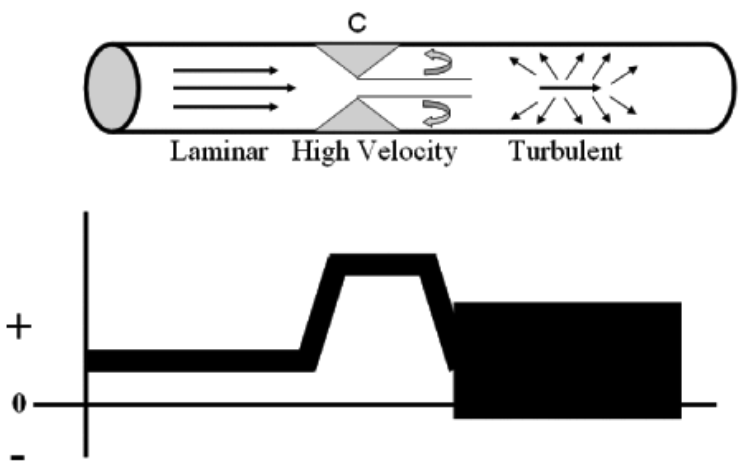

Fig. 1. Influence of constriction (C) on the blood velocities in a vessel (arrows indicate flow directions) and ranges of velocities at each cross-section along the vessel [1]

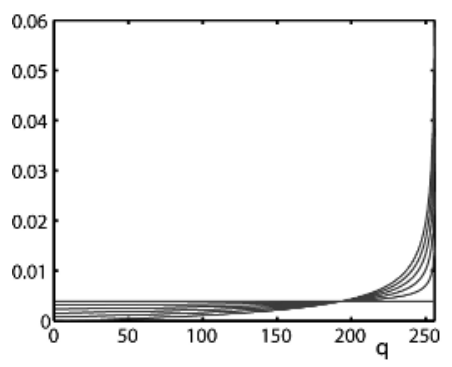

(a)

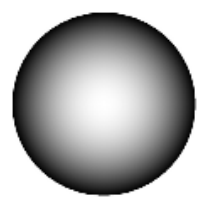

(b)

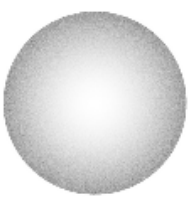

(c)

Fig. 2. Probability densities for Eq. (4) with $\beta=0.0,0.2,0.4, \ldots, 1.0$ and synthetic cross-section images of a blood vessel with laminar $(\beta=0, \mathrm{~b})$ and turbulent $(\beta=1$, c) flow

area for the circular vessel cross-section for radius $R$, respectively. Let $f\left(q \mid a_{r}\right)$, $\varphi_{\text {tur }}(q)$, and $\Phi_{\text {tur }}(x)=\operatorname{Pr}(q \leq x)=\int_{0}^{x} \varphi_{\text {tur }}(q) d q \Phi(x)$ denote the conditional density of intensities on the border of $a_{r}$, the unconditional density, and the probability of the intensities over the whole vessel, respectively.

The density of $y=\xi^{2} \in[0,1]$ is $p(y)=(2 \sqrt{y})^{-1}$ because $\operatorname{Pr}(y \leq x) \equiv$ $\operatorname{Pr}(-\sqrt{x} \leq \xi \leq \sqrt{x})=2 \sqrt{x}$. In accord with Eq. (1), $y=\frac{a_{\max }}{a_{r}}\left(1-\frac{q(r)}{q_{\max }}\right)$, and the intensities $q_{r} \in\left[q_{\max }\left(1-\frac{a_{r}}{a_{\max }}\right), q_{\max }\right]$ have the conditional density $f\left(q \mid a_{r}\right)=\frac{1}{2} \sqrt{\frac{a_{\max }}{a_{r} q_{\max }}} \frac{1}{\sqrt{q_{\max }-q}}$. The probability distribution of the intensities over the vessel area is then:

$$
\Phi_{\text {tur }}(x)=\frac{1}{a_{\max }} \int_{0}^{a_{\max }} d a_{r} \int_{0}^{x} f\left(q \mid a_{r}\right) d q=2-\frac{x}{q_{\max }}-2 \sqrt{1-\frac{x}{q_{\max }}}
$$

Therefore, the unconditional probability density is:

$$
\varphi_{\mathrm{tur}}(q)=\frac{1}{\sqrt{q_{\max }\left(q_{\max }-q\right)}}-\frac{1}{q_{\max }}
$$


Since the MRA may represent both normal and abnormal subjects, the model of vascular signals can be built as a mixture of the laminar and turbulent components:

$$
\varphi(q)=(1-\beta) \varphi_{\mathrm{lam}}(q)+\beta \varphi_{\mathrm{tur}}(q) \equiv \frac{1-2 \beta}{q_{\max }}+\frac{\beta}{\sqrt{q_{\max }\left(q_{\max }-q\right)}}
$$

Probability densities for different mixing weights $\beta \in[0,1]$ in this model are presented in Fig. 2.

\section{Adaptive Model of Multi-modal MRA}

MRA images contain three regions-of-interest (signal classes): $(i)$ darker CSF, bones and fat, ( $i$ i) brain tissues (gray matter and white mater), and (iii) brighter blood vessels. Marginal signal distributions for the first two classes are typically of intricate shape that differs much from the conventional individual Gaussians in 8 8 . The model in Eq. (4) describes only circular vessels and should have additional terms changing its shape to account for variations of the blood flow due to stenosis. Generally, no predefined probability model can accurately describe all the signal variations due to changes in blood velocity and viscosity, vessel diameter, and scanner sensitivity.

Therefore, we propose an adaptive probability model to handle both normal and abnormal MRA images. It mixes three submodels representing the abovementioned major image areas (abbreviated by "csf", "bt", and "bv", respectively):

$$
p_{\mathrm{MRA}}(q)=\sum_{i \in\{\mathrm{bv}, \mathrm{csf}, \mathrm{bt}\}} \alpha_{i} \varphi_{i}(q)
$$

where $\alpha_{i}$ are the mixing weights $\left(\alpha_{1}+\alpha_{2}+\alpha_{3}=1\right)$. Each of the three submodels $\varphi_{i}(q)$ is a mixture of one dominant component with a linear combination of several sign-alternate subordinate components chosen to closely approximate corresponding parts of an empirical marginal signal distribution $F_{\mathrm{emp}}=\left(f_{\mathrm{emp}}(q): q \in \mathbf{Q}\right)$.

The dominant component for the blood vessels submodel is the discrete parametric distribution $\Psi_{\theta, \mathrm{bv}}=\left(\psi_{\mathrm{bv}}(q \mid \theta): q=0, \ldots, q_{\max }\right)$ with a shorthand notation $\theta=\left(\beta, q_{\max }\right)$ for its parameters. It is obtained by integrating the density in Eq. (4) over unit intervals corresponding to the integer values $q \in \mathbf{Q}$ :

$$
\begin{aligned}
\psi_{\mathrm{bv}}(q \mid \theta) & =\frac{\int_{q}^{q+1} \varphi_{\mathrm{bv}}(q)}{q} d q \equiv(1-\beta) \frac{1}{q_{\max }+1} \\
& +\beta\left(2\left(\sqrt{1-\frac{q}{q_{\max }+1}}-\sqrt{1-\frac{q+1}{q_{\max }+1}}\right)-\frac{1}{q_{\max }+1}\right)
\end{aligned}
$$

Because typically $q_{\max }=Q-1$, this distribution has only a single parameter $\beta$.

Two other dominant components are discrete Gaussians (DGs) defined in 12 as a discrete probability distribution $\Psi_{\theta}=(\psi(q \mid \theta): q \in \mathbf{Q})$ integrating a normal parametric density over unit intervals: $\psi(q \mid \theta)=\Phi_{\theta}(q+0.5)-\Phi_{\theta}(q-0.5)$ for $q=1, \ldots, Q-2, \psi(0 \mid \theta)=\Phi_{\theta}(0.5), \psi(Q-1 \mid \theta)=1-\Phi_{\theta}(Q-1.5)$ where $\Phi_{\theta}(q)$ is 
the cumulative Gaussian probability function with parameters $\theta=\left(\mu, \sigma^{2}\right)$, i.e. the mean $\mu$, and variance $\sigma^{2}$.

The subordinate part of each submodel $\varphi_{i}(q)$ is a linear combination of discrete Gaussians (LCDGs) with $C_{i, \mathrm{p}}$ positive and $C_{i, \mathrm{n}}$ negative components under obvious restrictions on their weights. To identify the three submodels (estimate parameters of their dominant components and numbers and parameters of the positive and negative subordinate components), we use the EM-based techniques introduced in [12. The only difference here is the non-analytical estimation of the parameter $\beta$ on the M-steps using the gradient-based search for the global maximum of the goal likelihood function $G(\beta)=\sum_{q=0}^{q_{\max }} \pi(i=\operatorname{bv} \mid q) f_{\mathrm{emp}}(q) \ln \psi_{\mathrm{bv}}(q \mid \beta)$ where $\pi(i \mid q)$ is the responsibility of the submodel $i$ for $q$.

For simplicity, we do not restrict our model to only a proper subset of the LCDGs that ensures non-negative signal probabilities. This restriction may be ignored due to very close approximation provided by the model.

\section{Segmentation of Blood Vessels}

To justify the adaptive model of Eq. (5), Fig. 3 shows how different scanners effect the measurements. These three TOF-MRA slices were acquired for a subject with anemia using a Picker $1.5 \mathrm{~T}$ Edge MRI scanner with resolution of $512 \times 512 \times 93$, a subject with parietal lobe hemorrhage using a Signa Horizon GE 1.5T scanner with resolution $512 \times 512 \times 150$, and a normal subject using a state-of-art Siemens $3 \mathrm{~T}$ scanner with resolution $512 \times 512 \times 125$, respectively. The slice thickness is $1 \mathrm{~mm}$ in all the cases.

$\mathrm{A}$
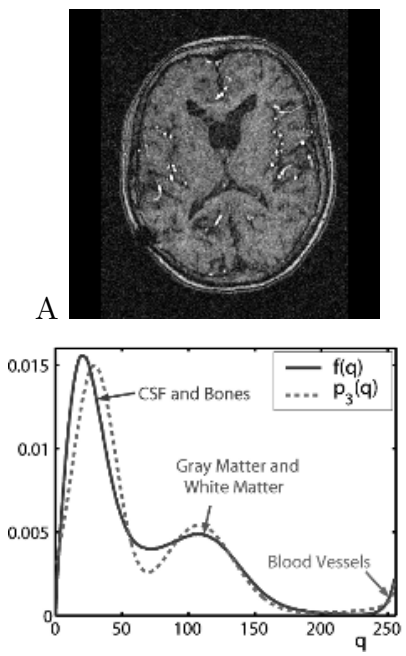

$\mathrm{B}$
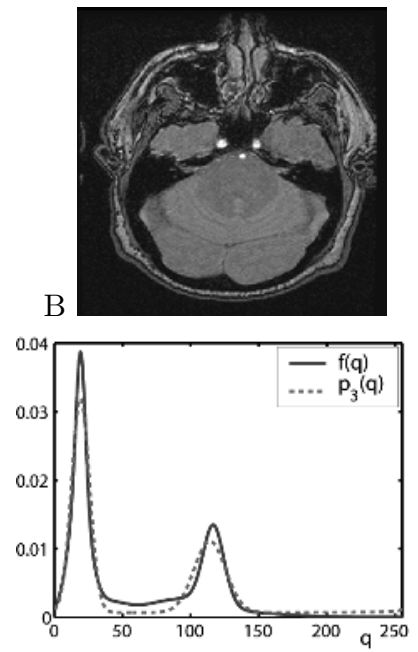
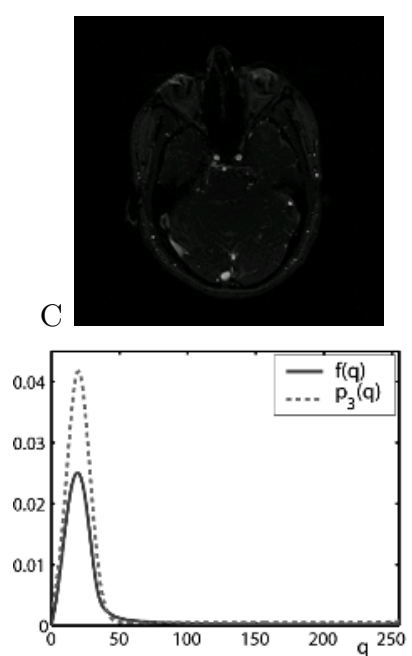

Fig. 3. Three TOF-MRA slices with their empirical distributions $f_{\text {emp }}(q)$ overlaid with the dominant mixtures $p_{3}(q)$ 

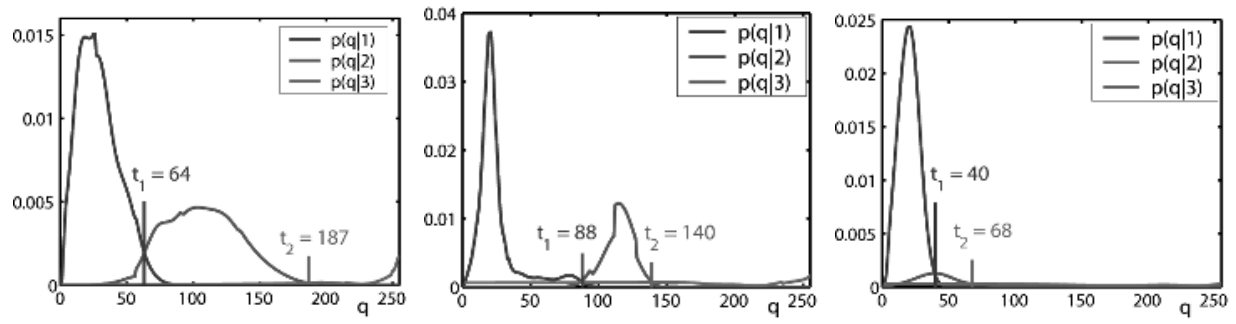

Fig. 4. Estimated marginal densities for the three classes MRA shown in Fig 3

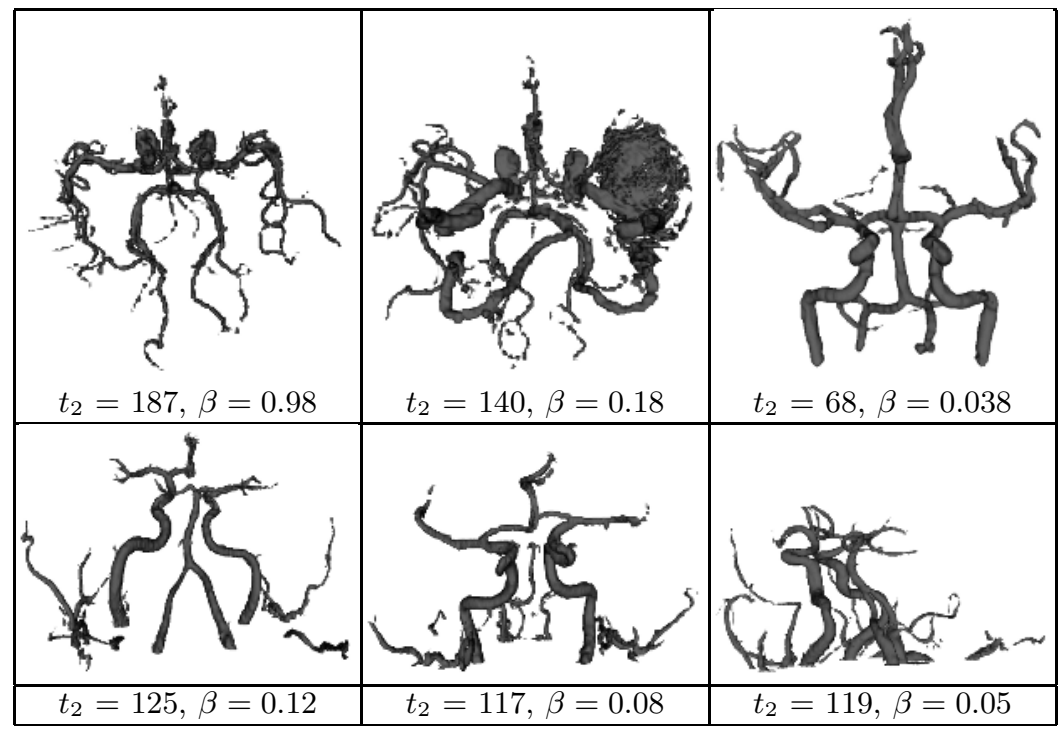

Fig. 5. Segmentation results of the proposed approach

The models of Eq. (5) were built with the EM-based approach (see [12] for detail). Figure 3 presents both the marginal empirical distributions $F_{\text {emp }}$ and the initial 3-component dominant mixtures for them containing the two Gaussian components and our model of blood vessels in Eq. (44). The estimated parameters $\beta$ of the latter are $0.92,0.18$, and 0.038 for the slices $\mathrm{A}$, $\mathrm{B}$, and $\mathrm{C}$ in Fig. 3, respectively, that reflects levels of blood turbulence expected from physics-based considerations. The second step of the proposed approach is to model the deviation between the empirical density and the three dominant modes, in this paper we used our previous modified EM algorithm [12] to perform this step, the final results of this approach are shown in Fig. 4. Figure 5 shows the segmentation results using the estimated densities shown in Fig. 4 . 


\section{Validation}

It is very difficult to accurately get manual segmented complete vasculare trees to validate our algorithm. To quantitatively evaluate its performance, we created three 3D phantoms in Fig. 6] with geometrical shapes similar to blood vessels with known ground truth. These three phantoms mimic bifurcations, zero and high curvature existing in any vascular system, and their changing radii simulate both large and small blood vessels. To make the distributions of these three phantoms similar to MRA images, first we compute the empirical class distributions $p(q \mid \mathrm{bv}), p(q \mid \mathrm{csf})$, and $p(q \mid \mathrm{bt})$ from the signals that represent blood vessels, CSF, and brain tissues from the MRA images segmented by a radiologist (we have selected 200 images from a data set of over 5000 images of 50 subjects). Then, The phantoms signal are generated by using the inverse mapping methods. The resulting phantom's histograms are similar to those in Fig. 3.

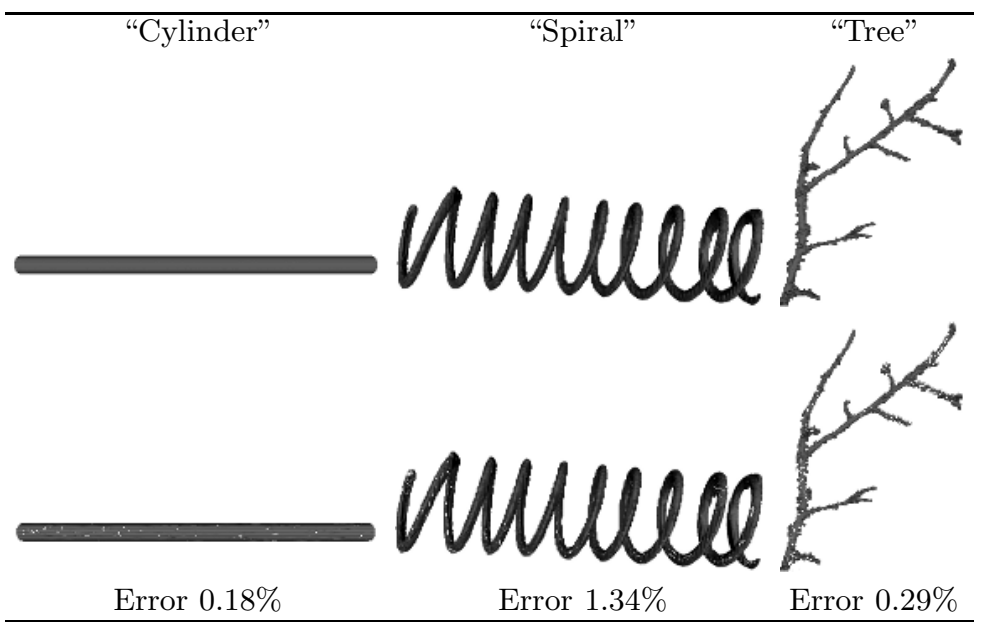

Fig. 6. Segmentation results of 3D phantoms (error shown in white color)

The total segmentation error is evaluated by a percentage of erroneous voxels with respect to the overall number of voxels in the ground truth $3 \mathrm{D}$ phantom. Figure 6] shows that, the maximum error obtained by the proposed approach is $1.34 \%$ and the minimum error is $0.18 \%$. These results confirm the high accuracy of the proposed approach.

Therefore, our adaptive model notably improves the accuracy of segmenting the MRA images acquired with different scanners. The conventional approaches either assume a purely laminar blood flow or pre-select a simple parametric distribution in attempts to take account of actual signal features. By contrast, our model is derived from the physical description of the blood flow and thus can accurately handle both normal and abnormal cases. Moreover, the estimated weights $\beta \in[0,1]$ in Eq. (4) provide a natural measure of the percentage of abnormality of the blood flow for a particular subject. 


\section{Conclusions}

We presented a new physically justified adaptive probability model of blood vessels on magnetic resonance angiography (MRA) images. It accounts for laminar (normal subjects) and turbulent blood flow (abnormal cases like anemia or stenosis). The high accuracy of segmenting MRA images with our approach is confirmed by experts-radiologists and also validated using special 3D geometrical phantoms.

Our present $\mathrm{C}++$ implementation of the algorithm on a single $2.4 \mathrm{GHz}$ Pentium 4 CPU with 512 MB RAM takes about 49 sec to segment 93 TOF-MRA slices of size $512 \times 512$ pixels each.

The proposed model is suitable for segmenting both TOF-MRA and PC-MRA images. Experiments with the latter type was not included in this paper due to space limitations.

\section{References}

1. T. McInerney and D. Terzopoulos, "Medical image segmentation using topologically adaptable surface," Proc. CVRMED-MRCAS'97, pp. 23-32, 1997

2. L. M. Lorigo, O. D. Faugeras, W. E. L. Grimson, and R. Keriven, "Curves: Curve evolution for vessel segmentation," Medical Image Analysis, vol. 5, pp. 195-206, 2001.

3. O. Wink, W. J. Niessen, and M. A. Viergever, "Fast delineation and visualization of vessels in 3-D angiographic images," IEEE Trans. Med. Imaging, vol. 19, pp. 337$346,2000$.

4. T. Deschamps and L. D. Cohen, "Fast extraction of tubular and tree 3D surfaces with front propoagation methods," Proc. $16^{\text {th }}$ ICPR, pp. 731-734, 2002.

5. P. Yan, A. A. Kassin, "MRA images segmentation with capillary active contour," Proc. MICCAI'05, pp. 51-58, 2005.

6. A. F. Frangi, W. J. Niessen, R. M. Hoogeveen, T. van Walsum, M. V. Viergever, "Model-based quantitation of 3-D magnetic resonance angiographic images," IEEE Transactions on Medical Imaging, vol. 18, pp. 946-956, 1999.

7. S. R. Aylward, E. Bullitt, "Initialization, noise, singularities, and scale in height ridge traversal for tubular object centerline extraction," IEEE Transactions on Medical Imaging, vol. 21, pp. 61-75, 2002.

8. D. L. Wilson and J. A. Noble, "An adaptive segmentation algorithm for time-offlight MRA data," IEEE Trans. Med. Imaging, vol. 18, pp. 938-945, 1999.

9. A. Chung, J. A. Noble, P. Summers, "Fusing speed and phase information for vascular segmentation of phase contrast MR angiograms," Medical Image Analysis, vol. 6, pp. 109-128, 2002.

10. C. G. Caro et al., The Mechanics of the Circulation, Oxford University Press, 1978.

11. W. F. Ganong, Review of medical physiology, McGraw-Hill,15th edition, 1991.

12. A. A. Farag, A. El-Baz, and G. Gimel'farb, "Precise Segmentation of Multi-modal Images," IEEE Transactions on Image Processing, vol. 15, no. 4, pp. 952-968, April 2006 . 